\title{
Effectiveness of frequency-lowering hearing aids and electric acoustic stimulation cochlear implant for treating people with a severe-to-profound high- frequency hearing loss
}

\begin{abstract}
Objectives: The objective of this research project was to compare the effectiveness of frequency-transposition, frequency-compression hearing aids and the electric acoustic stimulation (EAS) cochlear implant to improve speech recognition in participants with a sensorineural severe-to-profound high-frequency hearing loss (HFHL).

Design: Ten adults with a severe-to-profound HFHL were recruited. They were all tested with frequency-compression and frequency-transposition hearing aids following an $\mathrm{ABAC}$ single-subject design; four-week baselines were completed with their own hearing aids, followed by eight-week trials with each device. One participant also received an EAS implant after hearing aid trials. Follow-up time ranged from 16 to 32 weeks. Speech recognition was measured each week using sentence and monosyllable lists, in quiet and in noise. The subjective benefit with each technology was assessed with standardized questionnaires. Complementary data about the EAS implant effectiveness were also extracted from our database of EAS users.
\end{abstract}

Results: Frequency-lowering (FL) hearing aids improved speech recognition in five participants when compared to conventional hearing aids. Others experienced either no gain or some degradation in speech recognition when using a FL algorithm. Most participants reported better speech perception in everyday listening situations with FL hearing aids. Still, the participant who received an EAS implant obtained a greater improvement in speech recognition and reported a better benefit with this technology. Data collected from our database of EAS patients validated that the EAS participant was representative of our EAS users' population.

Conclusion: The EAS implant appears as the first indication for treating people with a severe-to-profound HFHL; it is also the costliest and most invasive alternative. Thus, and considering the significant benefit some participants obtained with FL hearing aids, trials using these technologies should be considered on an individual basis prior to implantation.

Keywords: hearing aids, frequency lowering, cochlear implants, electric acoustic stimulation, speech recognition, subjective benefit, high-frequency hearing loss, adults, recognition measures, $\mathrm{nfc}$ or $\mathrm{lft}$
Volume 6 Issue 3 - 2017

\author{
Mathieu Hotton, ${ }^{1,2,3}$ François Bergeron ${ }^{1,2}$ \\ 'Department of Rehabilitation Laval University, Canada \\ ${ }^{2}$ Centre for Interdisciplinary Research in Rehabilitation and \\ Social Integration, CIRRIS, Canada \\ ${ }^{3}$ Centre Intégré Universitaire de Santé et de Services Sociaux \\ (CIUSSS) de la Capitale-Nationale, Canada
}

\begin{abstract}
Correspondence: Mathieu Hotton, Department of Rehabilitation, Centre for Interdisciplinary Research in Rehabilitation and Social Integration, 525 Wilfrid-Hamel Blvd, room J-30.24, Québec City, Québec, GIM 2S8, Canada, Tel + I (418) 529-914I, Email Mathieu.hotton. I@ulaval.ca
\end{abstract}

Received: December 30, 2016 | Published: March 17, 2017
Abbreviations: APHAB, abbreviated profile of hearing aid benefit; CHU, centre hospitalier universitaire; CIRRIS, centre for interdisciplinary research in rehabilitation and social integration; CIUSSS, centre intégré universitaire de santé et de services sociaux; $\mathrm{CMR}$, centre montérégien de réadaptation; $\mathrm{CNC}$, consonantnucleus-consonant; CRIR, centre de recherche interdisciplinaire en réadaptation du montréal métropolitain; DSL, desired sensation level; EAS, electric acoustic stimulation; FL, frequency-lowering; FRQ-S, fonds de recherche du québec-santé; GHABP, glasgow hearing aid benefit profile; HA, hearing aid; HFHL, high-frequency hearing loss; HINT, hearing in noise test; IRD, institut raymond-dewar; LFT, linear frequency transposition; NFC, non-linear frequency compression; SNR, signal-to-noise ratio; TAM, test audiologique multimédia; TEN, threshold-equalizing-noise

\section{Introduction}

Frequency-lowering (FL) techniques have been developed and tested on people with a high-frequency hearing loss (HFHL) for many years. The objective being pursued with the use of this technology is to improve access to high-frequency sounds for hearing aid (HA) wearers. Actually, conventional HAs are known to be limited in their ability to deliver high levels of amplification in high frequencies, which may reduce the effectiveness of HAs, especially for people with a specific sensorineural severe-to-profound HFHL. ${ }^{1,2}$

Even if FL HAs have existed for a long time, the clinical diffusion remained limited until recently, when the development of digital HAs allowed for a more efficient implementation of FL algorithms. Nowadays, several makes and models of digital HAs feature FL

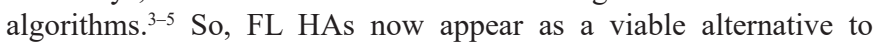
conventional amplification for people with a severe-to-profound HFHL.

Besides FL HAs, another technological alternative to conventional amplification for these persons arose from the field of cochlear implantation. The electric-acoustic stimulation (EAS) implant is a hearing device that combines a cochlear implant with an acoustic HA, allowing simultaneous transmission of low frequencies by conventional acoustic amplification and electric stimulation in high 
frequencies. ${ }^{6}$ Instead of shifting high frequencies to a lower frequency range, as it is done by FL HAs, the EAS implant uses electrical stimulation to convey high-frequency information. This technological advance, along with new electrode designs, improvements in surgical techniques and a reduction of implantation related risks opened the door of cochlear implantation to people with a specific severe-toprofound HFHL. ${ }^{7,8}$ Thus, those persons have nowadays access to a few technological alternatives to conventional HAs. However, the relative effectiveness of FL HAs and EAS implant to improve speech perception for this population is still not clear.

In a recent systematic review, Hotton \& Bergeron ${ }^{9}$ analyzed 80 research articles to determine which of frequency-transposition and frequency-compression HA, two types of FL, and EAS implant or conventional electric stimulation cochlear implant was the most effective to improve speech perception for people with a specific severe-to-profound HFHL. Results showed that the EAS implant was the most promising hearing technology for this population. Reported improvements with the EAS implant in comparison with conventional amplification were greater than for FL HAs or a conventional cochlear implant. However, the variability in the EAS implant effect reported across studies was important, illustrating that the potential benefit of the EAS implant may not be warranted for all individuals in the clinic. The effect of the conventional electric stimulation cochlear implant also appeared greater than the effect of FL HAs, the latter rendering smaller but still significant benefit to some individuals. No difference between the effectiveness of frequency-transposition and frequencycompression HAs was observed.

In the Hotton and Bergeron systematic review, ${ }^{9}$ comparisons between the effectiveness of FL HAs and the EAS implant had to be done indirectly, by comparing each technology to conventional HAs instead of comparing them directly to each other, since no published study did such comparison. This project addressed this issue by exploring this specific question: Among conventional, frequencycompression, frequency-transposition and electric acoustic stimulation hearing device, which technology is the most effective to improve speech recognition for people with a specific severe-to-profound sensorineural HFHL? Considering the reviewed scientific evidence, we hypothesized that speech recognition abilities of the participants 1) would be better with the EAS implant, and 2) would not be different with frequency-transposition or frequency-compression HAs.

\section{Materials and methods}

Ten adults with a bilateral severe-to-profound sensorineural HFHL with aidable residual hearing in low frequencies completed the study (six women and four men). They were all tested with nonlinear frequency compression (NFC; Phonak Naída Q70 SP or UP, or Naída III SP; Stäfa, Switzerland) and linear frequency transposition (LFT; Widex Dream 440 Fashion, Fusion P or Fusion HP; Lynge, Denmark) HAs following a prospective multicentric ABAC singlesubject design; four-week baselines were completed with their own hearing aids, followed by eight-week trials with each device. One participant also received an EAS implant (Med-El Concerto Mi1000 PIN with a Flex 24 electrode and a Sonnet EAS speech processor) after HA trials. Follow-up time ranged from 16 to 32 weeks. Participants were all considered as potential candidates for an EAS implant Unaided audiometric data, along with results to the ThresholdEqualizing-Noise (TEN) test, ${ }^{10,11}$ which was administered to detect the likelihood of a cochlear dead region, are presented in Table 1.

Participants were aged between 52 and 74years old. The duration of their deafness ranged from 11 to 61years, and they had a previous experience with conventional HAs of at least nine years. Canadian French was the first language of nine participants; the other one was English speaking. The research project had been approved by ethics boards of the Centre Hospitalier Universitaire (CHU) de Québec (\#2014-1860, C13-12-1860), of the Centre Intégré Universitaire de santé et de services sociaux (CIUSSS) de la Capitale-Nationale (\#2013-328) and of the Centre de Recherche interdisciplinaire en réadaptation du Montréal métropolitain (CRIR ; \#846-0513).

HAs were fitted according to the amplification targets prescribed by the Desired Sensation Level (DSL) 5.0a method, ${ }^{12}$ within a margin of $\pm 5 \mathrm{~dB}$. Fittings were confirmed by real-ear measurements and aided pure-tone thresholds. Two amplification programs were created in FL HAs, one with FL activated and a second with FL deactivated. All other features of the HAs (i.e. digital noise reduction, directional microphones, feedback cancellation, volume control, etc.) were left activated. FL parameters were individualized, using online fitting assistants from Alexander ${ }^{13}$ and the fitting protocol for FL HAs suggested by Scollie et al., ${ }^{14}$ In cases of bilateral fittings, FL parameters were set according to the hearing level of the best ear. HA models and FL fitting details for each participant are presented in Table 2. The speech processor of the participant who received an EAS implant was programmed as follows: FS4-P coding strategy, frequency allocation between 500 and $8400 \mathrm{~Hz}$, cut-off frequency for the acoustic part of the processor at $500 \mathrm{~Hz}$. Electrode insertion was complete, but 10 electrodes were activated because of non-auditory stimulation on electrodes 11 and 12 .

Testing was conducted by certified audiologists in three different clinics. Participants came to the clinic each week for speech recognition measures. Five speech perception tasks were assessed: ${ }^{15}$ Sentence recognition in quiet (in \%), sentence recognition in noise at fixed $+10,+5$ and $0 \mathrm{~dB}$ SNR (in \%), and monosyllable recognition in quiet (in \%). Sentence recognition in quiet and in noise was measured with the Canadian French and the American English versions of the Hearing in Noise Test (HINT). ${ }^{16,17}$ Sentences were presented in freefield at a fixed level of $63 \mathrm{dBA}$, and the noise level was varied between lists to obtain the desired SNR. Both sentences and background noise were presented via the same speaker. At each session, one list of 20 sentences was presented to the participant in each testing condition. Monosyllabic word recognition in quiet was assessed with Canadian French monosyllable lists from Benfante et al., ${ }^{18}$ and the CNC Word Test, ${ }^{19}$ in free-field, at a fixed presentation level of $60 \mathrm{~dB}$ HL. At each session, one list of 50 words was presented to the participant in eachtesting condition. All testing was done in an audiometric sound booth. Sentence and word lists and task presentation order was randomized. No sentence or word list was repeated in a same session. The Glasgow Hearing Aid Benefit Profile (GHABP) ${ }^{20}$ and the Abbreviated Profile of Hearing Aid Benefit (APHAB) ${ }^{21}$ were completed after each experimental phase to collect participants' perspectives on the benefits of each technology.

Speech recognition data were first analyzed on an individual basis, using a visual analysis method. ${ }^{22,23}$ Results to each test session were graphed, by outcome, and separately for each participant. An illustration of this individual visual analysis is presented in Figure 1. A $95 \%$ confidence interval was computed around the mean of the second baseline and was used as a reference for performance comparison between technologies (Figure 1, panels A \& B). This choice was made to avoid a task learning effect, as participants' performance during baseline 2 appeared generally better and more stable over time than during baseline 1 . Then, a proportion comparison statistical testing, called "prop. Test" function in the R statistical suite, ${ }^{24}$ was 
done to determine if the performance with FL HAs, in activated and deactivated FL conditions, or EAS implant was significantly different from the performance with conventional HAs at the second baseline. If a significant difference was found, the effect of FL HAs or EAS implant was computed by subtracting the mean of the second baseline to the mean of the concerned experimental phase. Performances with FL HAs in activated and deactivated FL conditions were also compared to each other using a similar procedure (Figure 1, panel C). Data from questionnaires were also analyzed individually, using published within-subject critical differences of the APHAB questionnaire ${ }^{21}$ and GHABP's norms. ${ }^{25}$ Then, data from all participants were grouped by technology. Friedman's non-parametric analyses of variance for repeated measures and Wilcoxon signed-rank tests with Bonferroni adjustments were computed on speech recognition, APHAB and GHABP mean group scores. For both individual and group analyses, the significance level was 0.05 ; a non-parametric approach to statistical analysis was chosen, because assumptions for parametric analysis were not met (i.e. non-normal distribution, non-independence of observations, small sample size).
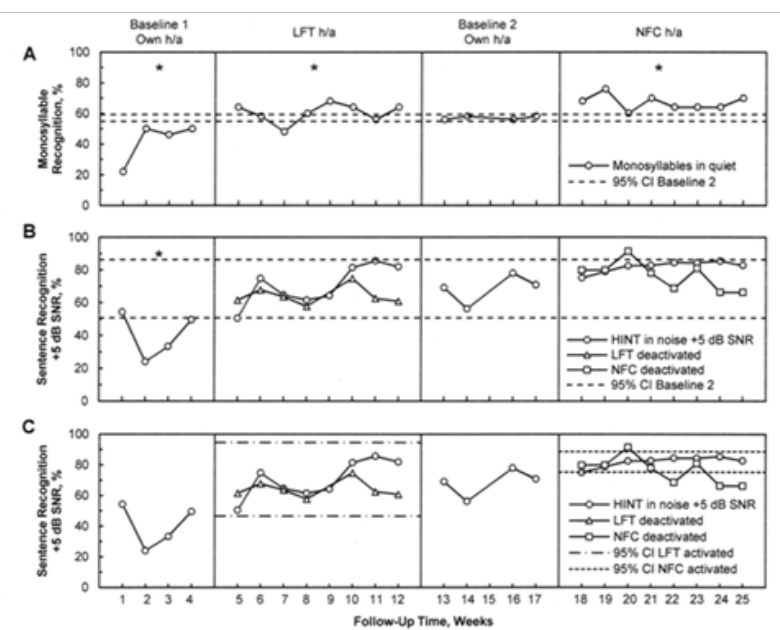

Figure I Results for the monosyllable recognition in quiet task (panel $A$ ), for the sentence recognition in noise at $+5 \mathrm{~dB}$ SNR (panels $B$ and $C$ ) for participant SI I, across the complete 25-week follow-up. Statistically significant differences are indicated by an asterisk (*: $p<0.000 \mathrm{I})$.

Cl: Confidence Interval; h/a: Hearing Aid; HINT: Hearing in Noise Test; LFT: Linear Frequency Transposition; NFC: Non-Linear Frequency Compression; SNR: Signal-To-Noise Ratio.

\section{Results}

A summary of statistically significant individual effects to speech recognition outcomes $(\mathrm{p}<.05)$ is presented in Table 3 . Detailed individual effects by participant are presented in Appendix A (Tables A1-A5). At an individual level, significant improvements in speech recognition scores with NFC HAs were noted for four participants, in comparison with conventional HAs. The observed gain ranged from 4.4 to $10.0 \%$ across outcomes. However, after comparing NFC on vs off conditions, only a small portion of this gain could be attributed to the activation of NFC (0.4 to $1.8 \%)$. Negative effects were also observed with NFC HAs for two participants, in comparison with conventional HAs. The decrease in performance was about -6.0 to $-5.0 \%$, and a portion of -5.0 to $-0.9 \%$ could be attributed to the activation of NFC. For LFT HAs, significant improvements in speech recognition scores were noted for four participants, in comparison with conventional HAs. The observed gain ranged from 3.3 to $8.7 \%$ across outcomes. After comparing LFT on vs off conditions, this gain could not be attributed to the activation of LFT for any participant. Negative effects were also observed with LFT HAs for four participants, in comparison with conventional HAs. The decrease in performance ranged from -22.1 to $-7.5 \%$, and a portion of $-15.5 \%$ could be attributed to the activation of LFT for one participant. Thus, for NFC and LFT HAs, the observed positive or negative effects may not be the result of the activation of FL alone, but of a more global technological effect, as a newer HA technology including FL, among other features (i.e. digital processor, noise reduction, microphones, feedback cancellation, etc.), was compared to an older one without FL. The participant who subsequently received an EAS implant performed significantly better with this technology, in comparison with conventional and FL HAs. Speech recognition scores were improved from 17.2 to $41.2 \%$ across outcomes. There was no negative effect on speech recognition with the EAS implant.

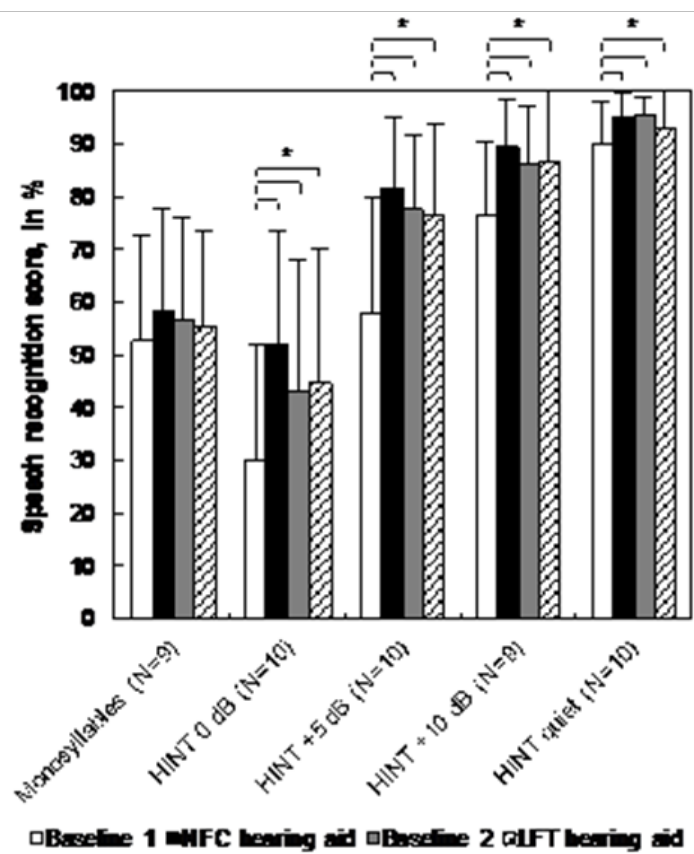

Figure 2 Group results for the speech recognition tasks with conventional (baselines I and 2), NFC and LFT HAs. Statistically significant differences are indicated by an asterisk $(*=p<0.0083)$.

LFT: Linear Frequency Transposition; NFC: Non-Linear Frequency Compression; HINT: Hearing in Noise Test; N: Number of Participants

Data from the GHABP showed that eight participants reported a significant decrease in their hearing disability with NFC and LFT HAs, in comparison with conventional amplification $(\mathrm{p}<.05)$. From the APHAB, two participants reported a significant decrease in their hearing disability with NFC HAs and two other participants with LFT HAs $(\mathrm{p}<.05)$. Two participants did not report a significant benefit with FL HAs in any of the two questionnaires. The participant who got an EAS implant reported a significant decrease in hearing disability with this technology to the two questionnaires $(\mathrm{p}<.05)$. Detailed individual results to the GHABP and APHAB questionnaires are presented in Appendix A (Table A6).

Group results to speech recognition outcomes for conventional, NFC and LFT HAs are shown in Figure 2. For sentence recognition in quiet and in noise outcomes, there was an overall significant difference between baselines 1 and $2(p<.0083)$, which supports the choice of baseline 2 as a reference for performance comparison between technologies. There was no significant group difference 
between baseline 2, NFC and LFT to any sentence recognition task $(\mathrm{p}>$.0083). There was a tendency towards a significant advantage of NFC over baseline 2 for sentence recognition in noise outcomes, but this advantage became nonsignificant after a Bonferroni adjustment $(.008<\mathrm{p}<0.05)$. For monosyllable recognition in quiet, there was no significant group difference between baselines 1 and 2, NFC and LFT.

Group results to sentence recognition in quiet, in noise at $+5 \mathrm{~dB}$ SNR and in noise at $0 \mathrm{~dB}$ SNR for NFC on vs off and LFT on vs off conditions are presented in Figure 3. For LFT HAs, a significant group difference was observed in noise at $0 \mathrm{~dB}$ SNR $(\mathrm{p}=.016)$. Thus, the group performance with LFT HAs was poorer with FL activated than with FL deactivated for this outcome. There was no significant group difference between LFT on and off conditions for the other outcomes. For NFC HAs, there was no significant group difference between NFC on and off conditions to any sentence recognition outcome $(\mathrm{p}>.05)$.

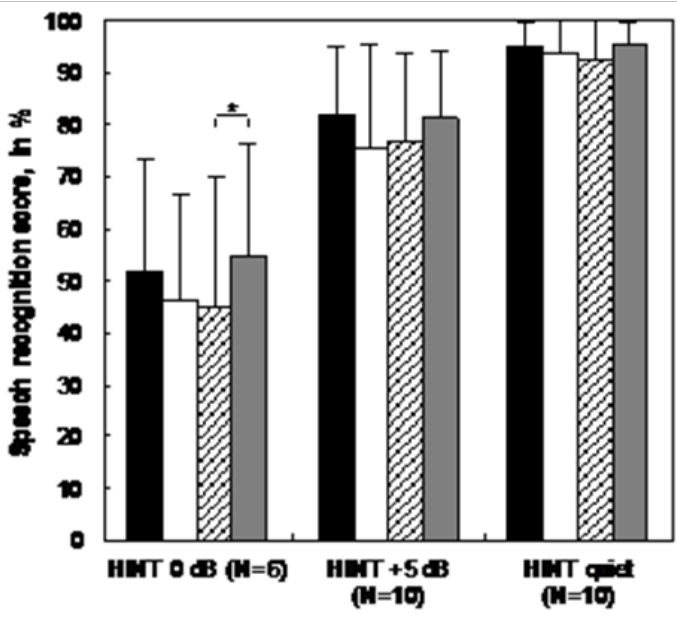

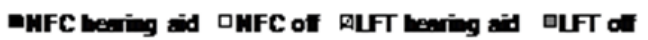

Figure 3 Group results for the speech recognition tasks, for the comparison between NFC and LFT HAs in FL activated and deactivated conditions. Statistically significant differences are indicated by an asterisk $(*=p=0.016)$.

LFT: Linear Frequency Transposition; NFC: Non-Linear Frequency Compression; HINT: Hearing in Noise Test; N: Number of Participants

Group results to the GHABP and APHAB questionnaires are presented in Figure 4 \& 5 respectively. For the GHABP, the mean group global hearing disability score was significantly lower with NFC and LFT in comparison with conventional HAs $(p<.017)$. The noise subscale score was also significantly lower with NFC in comparison with conventional HAs $(\mathrm{p}<.017)$. For the APHAB, the mean group global hearing disability score was significantly lower with LFT in comparison with conventional HAs $(\mathrm{p}<.017)$; there was also a tendency towards a significant advantage of NFC over conventional HAs, but this advantage became nonsignificant after a Bonferroni adjustment $(.017<\mathrm{p}<0.05)$. The background noise subscale score was also significantly lower with NFC in comparison with conventional HAs $(\mathrm{p}<.017)$. There was a tendency towards a significant advantage of LFT over conventional HAs for the ease of communication subscale, but this advantage became nonsignificant after a Bonferroni adjustment $(.017<\mathrm{p}<0.05)$. No difference was observed between NFC and LFT in any GHABP or APHAB subscale.

In order to determine if the EAS participant was representative of our EAS users' population, complementary data on EAS implant effectiveness were extracted from our database of EAS users (see Appendix B for details). Data collected with the HINT in quiet and in noise at fixed $+10,+5$ and $0 \mathrm{~dB}$ SNR, monosyllable lists from Benfante et al. and the Test Audiologique Multimédia (TAM) ${ }^{26}$ in quiet were included. Individual scores of the EAS participant were compared to $95 \%$ confidence intervals computed around the group mean scores of other EAS users $(\mathrm{N}=10)$ on these outcomes. The EAS participant's performance in the present study was considered similar to the group performance extracted from the database when its individual score fell inside the group 95\% confidence interval. Results of this analysis suggest that the performance of participant $\mathrm{S} 3$ appears representative of the performance of other EAS users in preimplantation for all assessed outcomes, but also in post-implantation, at least for outcomes and evaluation contexts less prone to training effect.

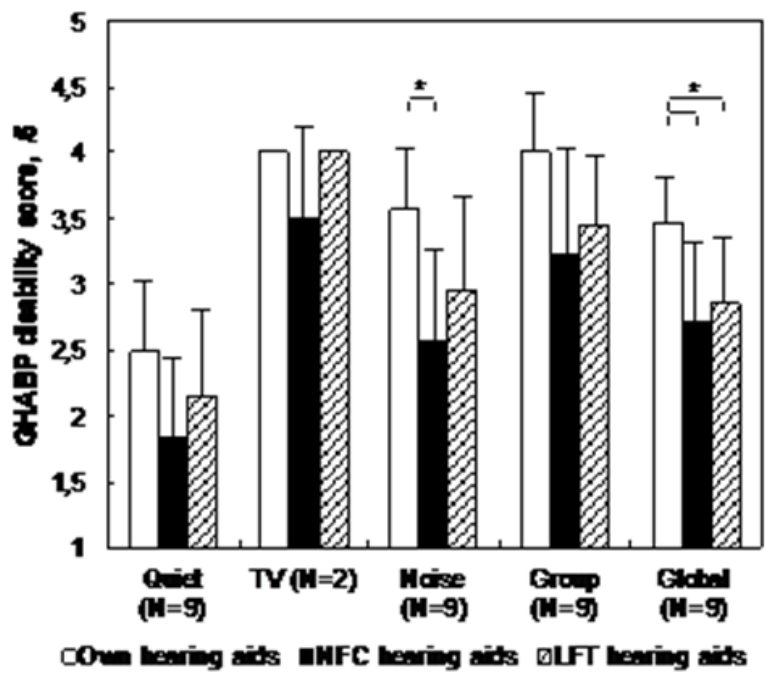

Figure 4 Group results for the GHABP questionnaire disability subscale. Statistically significant differences are indicated by an asterisk $(*=p<0.017)$

GHABP: Glasgow Hearing Aid Benefit Profile; LFT: Linear Frequency Transposition; NFC: Non-Linear Frequency Compression; HINT: Hearing in Noise Test; N: Number of Participants

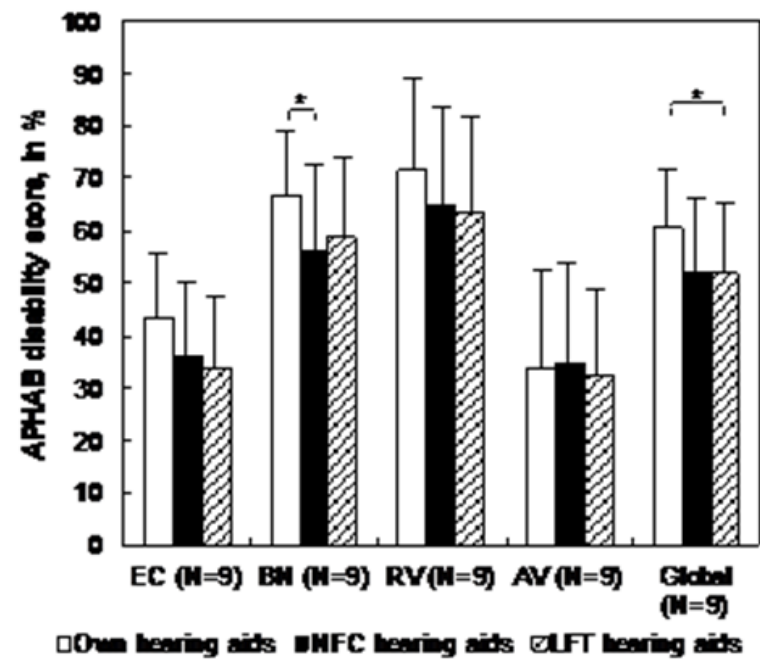

Figure 5 Group results for the APHAB questionnaire. Statistically significant differences are indicated by an asterisk $(*=p<0.017)$.

APHAB: Abbreviated Profile of Hearing Aid Benefit; LFT: Linear Frequency Transposition; NFC: Non-Linear Frequency Compression; N: Number of Participants 


\section{Discussion}

The objective of this research project was to determine which of NFC / LFT HAs and EAS cochlear implant is the most effective to improve speech recognition for people with a specific severeto-profound sensorineural HFHL. We hypothesized that speech recognition would be better with the EAS implant, and that speech recognition would not be different between LFT and NFC HAs.

Results obtained with participant S3 show that the EAS implant had a positive effect of up to $41.2 \%$ in speech recognition compared to conventional and FL HAs. For her, there was no advantage to use FL HAs in place of her conventional HAs, as the use of NFC and LFT HAs affected negatively her speech perception abilities. Clearly, the EAS implant appeared to be more effective for this participant Results to the GHABP and APHAB questionnaires supported these observations. As only one participant finally got a cochlear implant during the project, generalization on the EAS implant effectiveness is limited. Nevertheless, participant S3 performance was shown to be representative of other EAS users in our database; thus, it can be assumed that candidates with the same clinical profile (i.e. comparable audiometric thresholds and speech recognition scores before implantation) would probably benefit more from an EAS implant than from a FL HA. This would confirm our first hypothesis.

Frequency-lowering HAs also had a positive but smaller effect on speech perception for five participants, that was up to $10 \%$ with NFC HAs and up to $8.7 \%$ with LFT HAs. However, only a small part of this effect (up to $1.8 \%$ with NFC HAs and up to $2.7 \%$ with LFT HAs) could be attributed specifically to FL algorithms. Five other participants did not improve their speech recognition abilities with any of the NFC or LFT HAs. At a group-level, there was no significant improvement when using FL in comparison with conventional HAs, but a tendency towards an advantage of NFC HAs for sentence recognition in noise, with a mean score improvement ranging from 3.3 to $8.7 \%$, was noted. Thus, from an individual perspective, both NFC and LFT HAs could provide some gain for speech recognition in comparison with conventional HAs; this confirms our second hypothesis. Globally, results obtained on the effectiveness of the EAS implant, NFC and LFT HAs are in accordance with the conclusions of the systematic review from Hotton \& Bergeron ${ }^{9}$ where this comparison was realised indirectly.

When compared to the data previously reported in the literature, the observed effects in this study for FL strategies appear small. Indeed, for monosyllable recognition in quiet, Nyffeler ${ }^{27}$ reported a group mean gain with NFC HAs ranging from 17.5 to $40 \%$; with LFT HAs, Smith et al., ${ }^{28}$ reported a mean gain of $9.1 \%$ and individual effects of up to $19 \%$, and Kuk et al., ${ }^{29}$ mentioned a case where the gain with LFT HAs was $14 \%$. However, participants of those studies had better mean hearing thresholds than the present study. From participants with more comparable hearing levels, but fitted with an older type of frequency-compression, Miller-Hansen et al., ${ }^{30}$ observed a mean gain of $12.7 \%$ for word recognition in quiet, with individual effects up to $34 \%$. With the same technology, Parent et al., ${ }^{31}$ reported a mean gain of $20 \%$ for sentence recognition in quiet, with individual effects up to $37 \%$. In those two studies, the participants aided thresholds were generally better with the assessed frequency-compression device than with conventional HAs, not only for high frequencies, but also in lower frequencies, which was not the case in this study.

In the present study, the measured effects for FL strategies are so small that their clinical significance appears marginal. Considering that a change of 15 to $20 \%$ in the performance of an individual is generally expected to be considered clinically significant, ${ }^{32,33}$ most effects observed in this study would probably pass unnoticed in a standard clinical setting. But, even if the measured effects are small, benefits were reported by eight participants in GHABP and APHAB questionnaires. A majority of them judged their hearing disabilities reduced by the use of FL HAs in real-life. Moreover, statistically significant group differences were found in the GHABP global score, to the advantage of NFC and LFT HAs over conventional HAs, and for the APHAB global score, to the advantage of LFT HAs, with the difference almost reaching statistical significance for the NFC HAs. Also, NFC HAs showed a significant reduction on group hearing disability mean scores for GHABP listening in noise and APHAB background noise subscales. Therefore, small improvements in speech recognition offered by NFC and LFT HAs seem to be significant from the perspective of the participants.

There are at least a few explanations for the observed pattern of results. First, the speech recognition outcome measures we chose to assess the effectiveness of FL may not have been sufficiently sensitive. Learning and ceiling effects were observed for most participants, particularly for sentence recognition tasks. Learning effects were clearly visible for the majority of participants, as their performance during baseline 1 was poorer in comparison with baseline 2, showing a general improvement in performance over time. The measurement tools used may have been too repetitive or too easy for the study population, in the context of repeated measurements. These learning and ceiling effects may have imposed limits on the observable effect in this study. Also, selected measurement tools may not have been sensitive enough to the subtle effects of NFC or LFT HAs, or not representative enough of real life. Other researchers used more analytical tasks, such as consonant recognition or $/ \mathrm{s} /$ detection. ${ }^{34-37}$ We chose to use ecological outcome measures closer to real-life experience that are also considered clinical standard tests. Further research should be done to better understand the benefit of FL strategies and its relationship with speech recognition measures; this could help to select or develop more adapted measurement tools.

Secondly, there is a possibility that the observed pattern of results may be caused by a placebo effect. The placebo effect in HA research has been described in previously published papers. Bentler et al., ${ }^{38}$ reported that the labelling of a HA as "digital", in opposition to a "conventional" HA, led to an improvement of APHAB background noise and reverberation subscales' scores (5 to $9 \%$ ), even if the two assessed HA technologies were identical. No significant effect was observed on speech recognition scores, but $83 \%$ of the participants preferred the "digital" HA. In another study, ${ }^{39}$ the use of labels "new" vs "conventional" HA had a significant effect of $4.1 \%$ on speech recognition in noise; the sound quality of the "new" HA was systematically judged better, and was preferred by $75 \%$ of the participants. In those two studies, the proposed explanation for the observation of a placebo effect was that the "new" or "digital" labels raised participants' expectations about the assessed HA technologies, which subsequently impacted outcome measures. The results' pattern observed in those two placebo studies (i.e. small effects on behavioural speech recognition measures along with greater effects on subjective benefit, sound quality or preference ratings) was very similar to past unblinded HA comparison study results, ${ }^{40}$ and also to our present results.

In this study, we tried to avoid the placebo effect by using different methods. We provided as neutral as possible information to the participants about the assessed hearing technologies, before they entered the study, to minimize the effect of expectations. We used 
a single-subject design with two baselines and repeated measures over a prolonged period, to allow for within-subject control and variance calculations. During experimental phases with FL HAs, speech recognition measures with FL activated or deactivated were randomized without the knowledge of participants (i.e. single-blinded procedure). Then, the observed score change specifically due to FL activation is likely to reflect a true effect, but still, as not all procedures were blinded during the experimentation, the placebo effect cannot be completely ruled out. More research is needed to better understand how the placebo effect may show up in HA research and how to control for it, as the use of the ideal double-blinded randomized clinical trial is not always possible. Furthermore, a more in-depth exploration of the benefit of FL as experienced in real-life by the participants may help to enlighten the meaningfulness of the small improvements in speech recognition with FL that were observed in this study.

Noteworthy, we measured significant negative effects of FL HAs. At an individual level, these detrimental effects were as great as $6 \%$ for NFC HAs (up to 5\% attributable specifically to FL) and $22.1 \%$ for LFT HAs (up to $15.5 \%$ attributable specifically to FL). They were also more common with the latter technology. At a group level, there was a statistically significant difference between FL on and off conditions for LFT HAs, for sentence recognition in noise at $0 \mathrm{~dB}$ SNR, favouring the LFT deactivated condition. We observed that participants with a greater hearing loss between 1 and $2 \mathrm{kHz}$, with cochlear dead regions in the same frequency range, were those who got the most detrimental effects (ex. S3, S8 and S12). It may be because FL settings were stronger for them, the resulting distortion counteracting the potential benefits of the algorithm, or because of the severe cochlear damage in the destination frequency band. This suggests that there is a potential risk to degrade the patient's hearing abilities with the use of FL, even if the person reports a better benefit in real-life with this technology, as it was the case for three participants during this study (S3, S9 and S12). This risk may be greater with LFT HAs, as reported in previously published studies. ${ }^{41,42}$ Then, FL HAs may not be suitable for all patients with a HFHL, particularly those with a more severe hearing loss in mid frequencies.

In discussion to their systematic review, Hotton \& Bergeron suggested that trials with FL HAs before implantation might be a valuable tool in the context of candidacy assessments. However, these authors did not specify which FL algorithm should be used for this purpose. Unfortunately, results of this study do not help to answer this question. Both NFC and LFT HAs seem to have the potential to be beneficial to people with a HFHL, but there was no clear advantage for one algorithm versus the other.

\section{Conclusion}

EAS implant appears as the first indication for treating people with a high-frequency hearing loss. However, FL HAs can provide a significant benefit for some individuals. Even if the effect assessed with traditional speech recognition measures is small, it can be significant from the perspective of the patient. In this context, and considering the potential risks and high costs related to cochlear implantation, trials with FL should be considered on an individual basis prior to implantation.

\section{Acknowledgments}

The authors are very grateful to the participants, who took much of their personal time and invested themselves completely in the project. The authors also wish to thank the personnel from the Audiology Centre-West clinic in Montréal, the CIUSSS de la Capitale-Nationale in Québec City, and the Centre Montérégien de réadaptation (CMR) in Granby, for data collection, and also the CHU de Québec and Institut Raymond-Dewar (IRD) for help with recruitment. Finally, many thanks to Mr. Jean Leblond for statistical analysis counselling.

\section{Financial disclosures/conflicts of interest}

This research was supported by grants from the Fonds de Recherche du Québec en santé (FRQ-S), the CIUSSS de la Capitale-Nationale, and the Centre for Interdisciplinary Research in Rehabilitation and Social Integration (CIRRIS). Phonak Canada and Widex Canada provided HA loaners at no cost for the duration of the trial.

Appendix A: Individual Results to Speech Recognition Outcomes and Benefit Questionnaires.

Appendix B: Data Extracted From EAS Implant Database, Compared to Participant S3 Scores.

\section{Funding}

None.

\section{References}

1. Ching TY, Dillon H, Byrne D. Speech recognition of hearing-impaired listeners: predictions from audibility and the limited role of highfrequency amplification. J Acoust Soc Am. 1998;103(2):1128-1140.

2. Hogan CA, Turner CW. High-frequency audibility: benefits for hearingimpaired listeners. J Acoust Soc Am. 1998;104(1):432-441.

3. Alexander J. Individual variability in recognition of frequency-lowered speech. Semin Hear. 2013;34:86-109.

4. Kamilla Angelo, Joshua M Alexander, et al. Oticon Frequency Lowering. Access to high-frequency speech sounds with Speech Rescue technology. White Paper. 2015.

5. Simpson A. Frequency-lowering devices for managing high-frequency hearing loss: a review. Trends Amplif. 2009;13(2): 87-106.

6. Von Ilberg CA, Baumann U, Kiefer J, et al. Electric-acoustic stimulation of the auditory system: A review of the first decade. Audiol Neurootol. 2008;16(Suppl 2):1-30.

7. Gifford RH, Dorman MF, Shallop JK, et al. Evidence for the expansion of adult cochlear implant candidacy. Ear Hear . 2010;31(2):186-194.

8. Sampaio AL, Araujo MF, Oliveira CA. New criteria of indication and selection of patients to cochlear implant. Int $J$ Otolaryngol. 2011;2011:573968.

9. Hotton M, Bergeron F. A systematic review on the effectiveness of hearing technologies for people with a severe-to-profound highfrequency hearing loss. Can J Speech Lang Pathol Audiol. 2014.

10. Moore BC, Creeke S, Glasberg BR, et al. A version of the TEN Test for use with ER-3A insert earphones. Ear Hear. 2012; 33(4):554-557.

11. Moore BC, Glasberg BR, Stone MA. New version of the TEN test with calibrations in dB HL. Ear Hear. 2004;25(5): 478-487.

12. Scollie S, Seewald R, Cornelisse L, et al. The Desired Sensation Level multistage input/output algorithm. Trends Amplif. 2004;9(4):159-197.

13. http://web.ics.purdue.edu/ alexan14/fittingassistants.html

14. Scollie S, Glista D, Seto J, et al. Fitting Frequency-Lowering Signal Processing Applying the American Academy of Audiology Pediatric Amplification Guideline: Updates and Protocols. J Am Acad Audiol . 2016;27(3):219-236.

15. Advanced Bionics LLC, Cochlear Americas, MED-EL Corporation. New minimum speech test battery (MSTB) for adult cochlear implant users 2011: user manual. USA. 2011. 
16. Vaillancourt V, Laroche C, Mayer C, et al. Adaptation of the HINT (hearing in noise test) for adult Canadian Francophone populations. Int J Audiol. 2005; 44(6):358-369.

17. Nilsson M, Soli SD, Sullivan JA. Development of the Hearing in Noise Test for the measurement of speech reception thresholds in quiet and in noise. J Acoust Soc Am. 1994;95(2):1085-1099.

18. Benfante H, Charbonneau R, Arsenault A, et al. Audiométrie vocale. Listes de mots phonétiquement balancés. Hôpital Maisonneuve, Montréal, Canada. 1966.

19. Peterson GE, Lehiste I. Revised CNC lists for auditory tests. J Speech Hear Disord. 1962;27:62-70.

20. Gatehouse S. Glasgow Hearing Aid Benefit Profile: derivation and validation of a client-centered outcome measure for hearing aid services. J Am Acad Audiol. 1999;10:80-103.

21. Cox RM, Alexander GC. The abbreviated profile of hearing aid benefit. Ear Hear. 1995;16(2):176-186.

22. Janosky JE, Leininger SL, Hoerger MP, et al. Single Subject Designs in Biomedicine. Springer, USA. 2009.

23. Leslie G Portney, Mary P Watkins. Foundations of Clinical Research : Applications to Practice. (3rd edn), Upper Saddle River, NJ, USA. 2009.

24. https://www.R-project.org

25. Whitmer WM, Howell P, Akeroyd MA. Proposed norms for the Glasgow hearing-aid benefit profile (Ghabp) questionnaire. Int J Audiol. 2014;53(5):345-351.

26. Bergeron F. Développement d'une batterie de tests multimédias visant à évaluer les habiletés audiovisuelles de perception de la parole. Can J Rehab. 1998;11(4):182-183.

27. Nyffeler M. The Naída Power Hearing Instrument Family-Field Test Results demonstrate better speech clarity-unparalleled in its class. 2008.

28. Smith J, Dann M, Brown M. An evaluation of frequency transposition for hearing-impaired school-age children. Deafness \& Education International. 2009;11(2):62-82.

29. Kuk F, Keenan D, Peeters H, et al. Critical factors in ensuring efficacy of frequency transposition part 1: Individualizing the start frequency. The Hearing Review. 2007.
30. Miller Hansen DR, Nelson PB, Widen JE, et al. Evaluating the benefit of speech recoding hearing aids in children. Am J Audiol. 2003;12(2):106-113.

31. Parent TC, Chmiel R, Jerger J. Comparison of performance with frequency transposition hearing aids and conventional hearing aids. $J$ Am Acad Audiol. 1998;9(1):67-77.

32. Thornton AR, Raffin MJ. Speech-discrimination scores modeled as a binomial variable. J Speech Hear Res. 1978;21(3):507-518.

33. Raffin MJ, Thornton AR. Confidence levels for differences between speech-discrimination scores. A research note. J Speech Hear Res. 1980;23(1):5-18.

34. Auriemmo J, Kuk F, Lau C, et al. Effect of linear frequency transposition on speech recognition and production of school-age children. $J$ Am Acad Audiol . 2009;20(5):289-305.

35. Glista D, Scollie S, Bagatto M, et al. Evaluation of nonlinear frequency compression: clinical outcomes. Int J Audiol. 2009;48(9):632-644.

36. Glista D, Scollie S, Sulkers J. Perceptual Acclimatization Post Nonlinear Frequency Compression Hearing Aid Fitting in Older Children. J Speech Lang Hear Res. 2012; 55(6):1765-1787.

37. Robinson JD, Stainsby TH, Baer T, et al. Evaluation of a frequency transposition algorithm using wearable hearing aids. Int $J$ Audiol. 2009;48(6):384-393.

38. Bentler RA, Niebuhr DP, Johnson TA, et al. Impact of digital labeling on outcome measures. Ear Hear . 2003;24(3):215-224.

39. Dawes P, Hopkins R, Munro KJ. Placebo effects in hearing-aid trials are reliable. Int J Audiol. 2013; 52(7): 472-477.

40. Dawes P, Powell S, Munro KJ. The placebo effect and the influence of participant expectation on hearing aid trials. Ear Hear. 2011;32(6):767-774.

41. Alexander JM, Kopun JG, Stelmachowicz PG. Effects of frequency compression and frequency transposition on fricative and affricate perception in listeners with normal hearing and mild to moderate hearing loss. Ear Hear. 2014;35(5): 519-532.

42. Miller CW, Bates E, Brennan M . The effects of frequency lowering on speech perception in noise with adult hearing-aid users. Int J Audiol. 2016;55(5):305-312. 